\title{
Long-Term Exposure to Ambient Air Pollution and Type 2 Diabetes in Adults
}

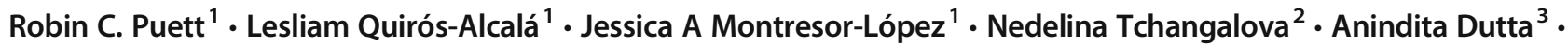 \\ Devon Payne-Sturges ${ }^{1}$. Jeff D. Yanosky ${ }^{4}$
}

Published online: 9 February 2019

(C) Springer Nature Switzerland AG 2019

\begin{abstract}
Purpose of Review We identified 24 publications from January 2010 until September 2018 in the peer-reviewed literature addressing the relationship of long-term air pollution exposures and type 2 diabetes-related morbidity and mortality among adults. We examine key methodological issues, synthesize findings, and address study strengths and limitations. We also discuss biological mechanisms, policy implications, and future research needed to address existing knowledge gaps.

Recent Findings In general, the studies included in this review employed rigorous methodology with large sample sizes, appropriate study designs to maximize available cohort study or administrative data sources, and exposure modeling that accounted for spatial patterns in air pollution levels. Overall, studies suggested increased risks of type 2 diabetes-related morbidity and mortality among adults associated with increased exposures; however, findings were not uniformly positive nor statistically significant.

Summary Current research is particularly limited regarding the biological mechanisms involved and the relationship between ozone and diabetes. Additionally, more research is needed to distinguish clearly the effects of nitrogen oxides from those of other pollutants and to identify potential subpopulations with greater susceptibility for certain pollutant exposures. A better understanding of the potential link between long-term ambient air pollution exposures and type 2 diabetes may provide opportunities for the reduction of health risks and inform future interventions for environmental protection and diabetes management.
\end{abstract}

Keywords Air pollution · Type 2 diabetes $\cdot$ Chronic exposures

This article is part of the Topical Collection on Environmental Epidemiology

Electronic supplementary material The online version of this article (https://doi.org/10.1007/s40471-019-0184-1) contains supplementary material, which is available to authorized users.

Robin C. Puett

rpuett@umd.edu

1 Maryland Institute for Applied Environmental Health, University of Maryland School of Public Health, College Park, MD, USA

2 STEM Library, University of Maryland, College Park, MD, USA

3 Department of Medicine, University of Chicago, Chicago, IL, USA

4 Division of Epidemiology, Department of Public Health Sciences, Pennsylvania State University College of Medicine, Hershey, PA, USA

\section{Introduction}

According to the World Health Organization, exposure to ambient air pollution is responsible for 4.2 million premature deaths worldwide every year $[1 \bullet]$. Ambient air pollution is a complex mixture of natural and synthetic substances, containing many chemical and biological constituents, in the form of gases and particulate matter released into and formed in the atmosphere. In addition to particulate matter, other major constituents of air pollution include ozone, nitrogen dioxide, sulfur dioxide, carbon monoxide, and lead [2]. Studies conducted over the last three decades have implicated exposure to ambient air pollution constituents to various adverse health effects, including cardiovascular disease, respiratory diseases, adverse pregnancy outcomes, cancer, and more recently, altered neurodevelopment [3-6]. Prior research has also shown that people with type 2 diabetes mellitus have higher risks of air pollution-related cardiovascular and respiratory outcomes compared with healthier populations $[7,8 \cdot \bullet, 9,10]$. There is 
also emerging evidence that increases in exposures to ambient air pollution may be associated with increased risks of type 2 diabetes morbidity and mortality among adults $[11,12]$.

The potential link between ambient air pollution and diabetes is of significant public health concern as the global health and economic burden from each of these is large and expected to increase. For example by 2030, diabetes is estimated to result in a global economic burden of $\$ 2.1$ trillion USD and is projected to be the seventh leading cause of death worldwide [13]. Diabetes is a chronic disease that results from the body's inability to produce the insulin, which helps regulate the amount of glucose in the blood. The most common types of diabetes are type 1 , type 2 , and gestational diabetes. Type 1 diabetes results when the body cannot produce any insulin, while type 2 results when the body does not produce sufficient insulin. Type 2 diabetes is the most common type of diabetes and occurs most often among middle-aged and older adults [14]. While the exact biological mechanisms by which ambient air pollutants could increase an individual's risk of developing diabetes have not been clearly elucidated, several mechanisms have been proposed including impaired glucose metabolism and inflammation [15-17]. In addition, an animal study conducted by Sun et al. suggests linkages between particulate matter exposure and insulin resistance, supporting biologic plausibility [18].

In this review article, we examine peer-reviewed epidemiologic studies published from January 2010 until September 2018 that evaluated the role of long-term ambient air pollution exposures in the risk of type 2 diabetes-related morbidity and mortality among adults. We examine key methodological issues, synthesize findings, and address study strengths and limitations. We also discuss biological mechanisms, policy implications, and future research needed to address existing knowledge gaps.

\section{Methods}

The US National Library of Medicine's Pubmed database was originally searched in March, 2018, and a more systematic search developed by an experienced academic Public Health Librarian was conducted on September, 2018 to verify and update the results.

The initial search strategy combined 'diabetes' as a Medical Subject Headings (MeSH) term and keyword with the following MeSH terms and keywords for air pollution: 'air pollution', 'particulate matter', 'particulates', 'nitrogen oxides', 'nitrogen dioxide', 'ozone', and 'traffic air pollution.' Initial searches used the broad term 'air pollution'; however, given the majority of resulting abstracts focused on the specific pollutants aforementioned and for the purposes of addressing the principal air pollutants considered in regulatory policies, we employed the more specific pollutant terms in order to capture additional abstracts that did not appear under the broad term. The large number of articles and initial manual review of all identified abstracts lead us to add the following keywords to further the search focus: 'long-term' and 'chronic'. We excluded studies with keywords or MeSH terms of 'smoking' and 'tobacco'. Using filters, we limited our search to adult populations (to focus on type 2 diabetes), full articles, original research, and human studies published in English between 2010 and 2018. A manual search of abstracts was conducted to identify epidemiologic studies with a main research question/hypothesis and analyses addressing the relationship between type 2 diabetes and long-term exposure to air pollution.

Consequently, a similar search strategy was developed by an experienced academic Public Health Librarian (Appendix 1) based on the MeSH terms and keywords frequently used in the 21 articles identified through the first search. A total of 432 references were initially identified followed by a manual process of selection and screening titles and abstracts (Appendix 2). Full text manual screening of the 31 selected records resulted in a final set of 24 publications of 21 study populations.

We note that most publications reported results from several statistical models (e.g., adjusted for different confounders, sensitivity analyses). In this review, we attempt to present at least one fully adjusted model from the main analyses; however, our review is not an exhaustive nor comprehensive discussion of all results for each publication. For example, if results were reported for exposures modeled using different strategies, we may not have included results from each strategy, particularly if results were comparable.

\section{Epidemiologic Study Design Strengths and Limitations}

The 24 publications in this review focused on 21 study populations and were conducted in Europe, North America, and China (Table 1); only a limited number of studies focused on highly exposed populations. Global representation is likely limited due to the databases searched and use of English as the publication language, but also potentially due to the uneven availability of air quality monitoring throughout the world. The majority of the selected publications used cohorts, including five cohorts exclusively enrolling women and one study exclusively enrolled men [17, 22•, 23, 24•, 29•, 35, 40]. Among those studies using a cohort design, about half had follow-up periods of approximately 10 years or longer, which is a major strength for identifying cases of incident diabetes $[20 \bullet, 21,22 \bullet, 23,24 \bullet, 29 \bullet, 34 \bullet, 35,37,40]$. A few publications were cross-sectional analyses of a health survey [27, 31, 39]. The studies reviewed generally had large sample sizes (ranging from 1775 to $2,145,400$ ), and as with many large epidemiologic studies, many of those included in our review 


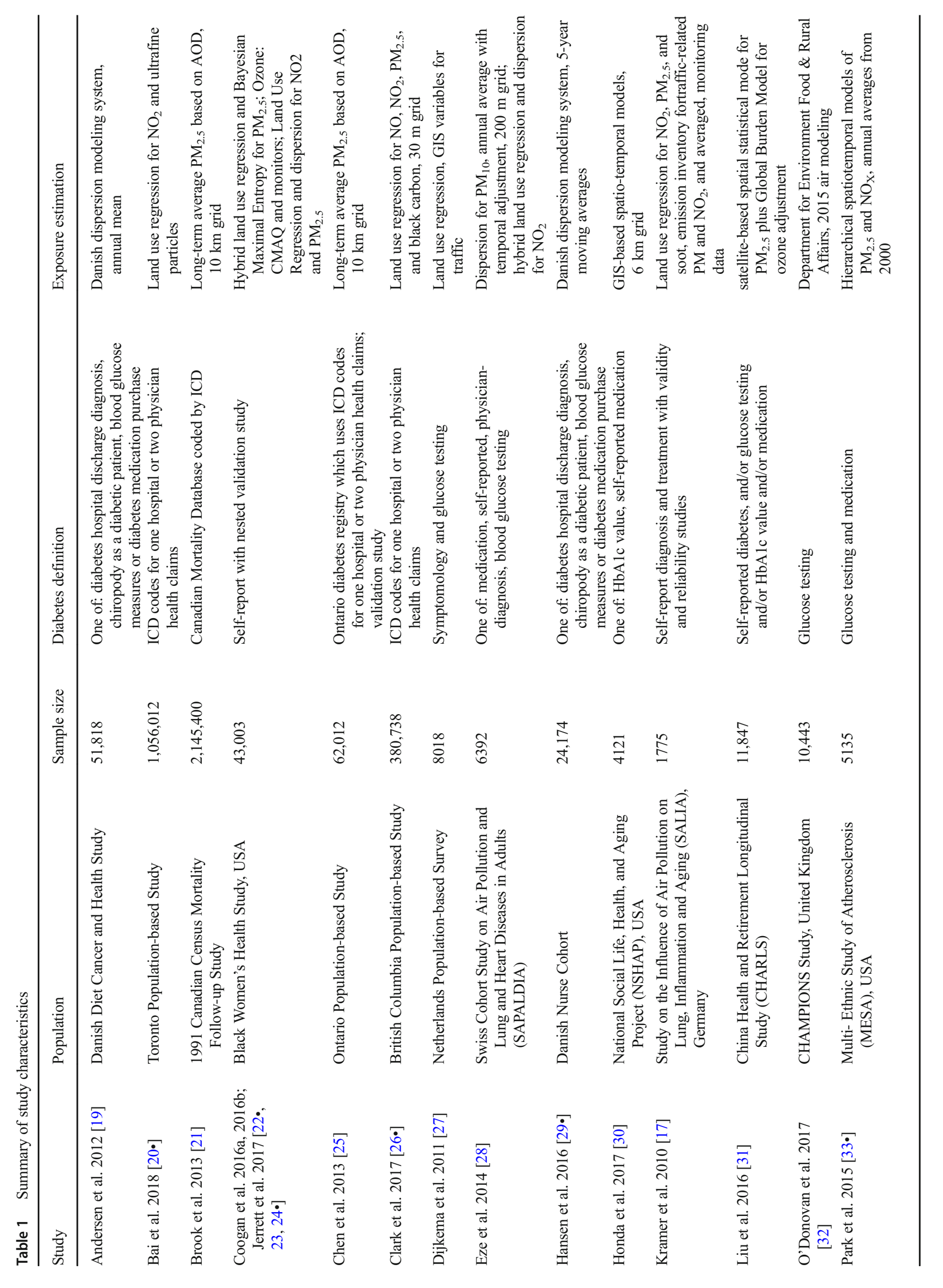




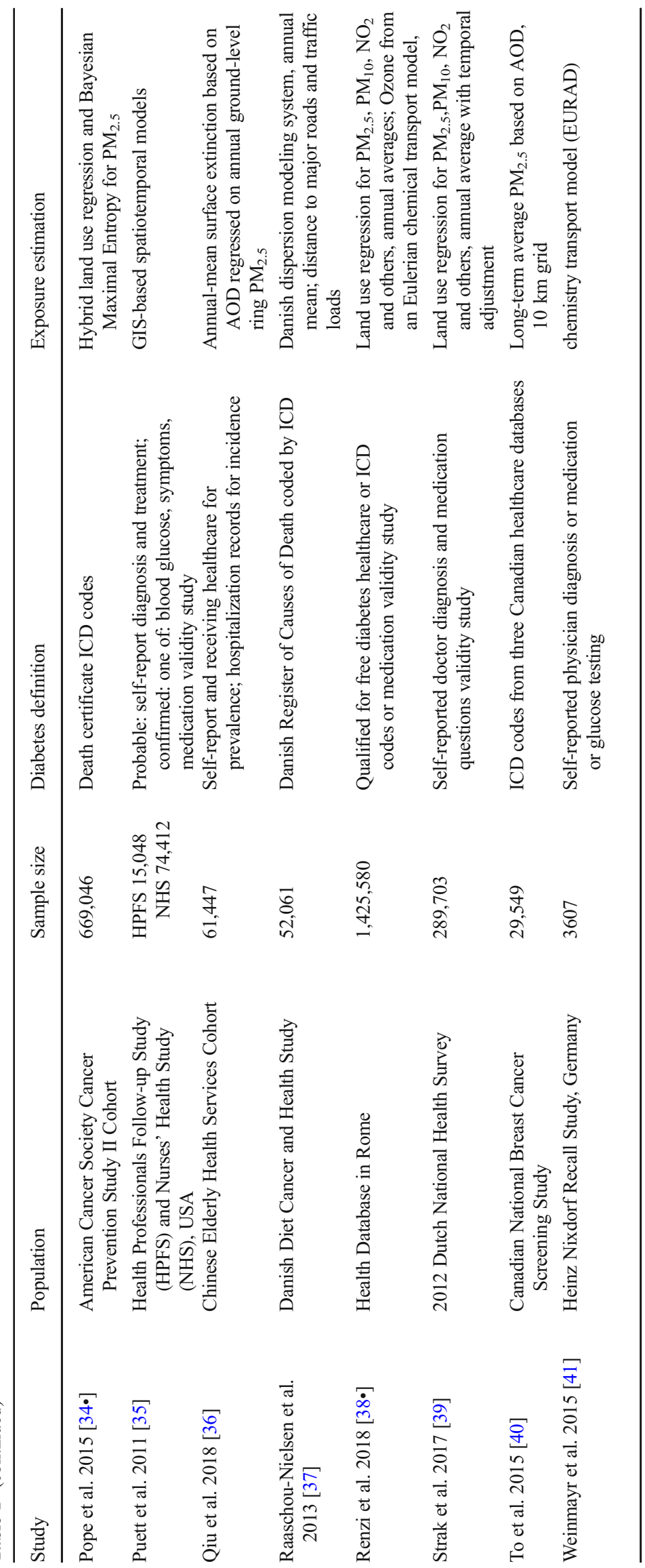


primarily used administrative data for defining type 2 diabetes (e.g., ICD codes from death certificates, hospitalization, or health care visits $[17,20 \bullet, 21,25,26 \bullet, 34 \bullet, 36,37,38 \bullet 40])$. While the use of administrative data has the potential of introducing bias due to misclassification of disease resulting from challenges inherent in health system coding, use of this type of data does offer higher power to detect effects due to the larger sample sizes these data afford.

Several other studies were survey-based, initially relying on self-reported physician diagnosis of diabetes. However, many of these studies employed additional methods in attempts to improve or examine diabetes assessment accuracy, including querying participants or health records for diabetes medication use and conducting nested validation studies [17, 22•, 23, 24•]. Other studies used a combination of information (e.g., treatment, biomarker measures, discharge diagnoses) and designated study participants as having diabetes if any of the information was suggestive of having the disease $[19,25,28,29 \bullet, 30$, $31,38 \cdot, 41]$. Many studies used information from glucose testing, one of the measures included in the World Health Organization and American Diabetes Association diagnostic criteria $[19,27,28,29 \bullet, 31,32,33 \bullet, 35,41 \bullet]$. To provide information about the level of accuracy for diabetes assessment and to facilitate translation of epidemiological research to clinical guidelines, a comparison of outcome definitions with national or international diagnosis criteria is beneficial [e.g., 27, $31,33 \cdot, 35,42]$. These standards require access to blood tests and symptomology reports, reflecting the challenges involved with obtaining specified laboratory testing (e.g., fasting for at least $8 \mathrm{~h}$ and using certified standardized assays) on specified criterion measures [43]. However, reliance on self-report or medical record report of physician diagnosis alone could lead to potential misclassification of disease given that the worldwide prevalence of undiagnosed type 2 diabetes has been estimated between 45 and 50\% [44, 45]. Related to this, comparisons of self-report to biomedical measures tend to show low sensitivity (55-80\%), but high specificity (84-98\%) [46, 47]. Though self-report of physician diagnosis alone is typically highly specific, a study using information from a US cohort on atherosclerosis observed the magnitude of associations between known risk factors and self-reported diabetes were attenuated compared to using case definitions which included one or two of the following criteria: self-report, glucose testing, or medication. Differences in the strength of associations when defining diabetes with one versus two of the criteria were inconsistent [48].

\section{Exposure Assessment}

Overall, the majority of studies relied on exposure estimates from various modeling strategies that included dispersion factors such as land use and meteorology. If both particulate matter and nitrogen dioxide $\left(\mathrm{NO}_{2}\right)$ or nitrogen oxides were considered in the same study, investigators tended to use the same modeling strategy to estimate both exposures. However, some studies also included an additional model for one of the pollutants. For example, some studies used dispersion and land use regression modeling [22•,28]. Generally, dispersion and spatiotemporal modeling were used to estimate particulate matter and $\mathrm{NO}_{2}$ or nitrogen oxides exposures for cohort or national studies that had access to participant's residential addresses. These studies benefit from more highly spatially resolved information on air pollution exposure assessment as compared with the use of nearest monitors $[19,22 \cdot, 23,28$, $29 \bullet, 33 \cdot, 35,37]$. Spatiotemporal modeling was applied mostly in cohort studies that covered a range of cities/regional areas in the USA. The method was used to estimate $\mathrm{NO}_{2}$ or nitrogen oxides and particulate matter exposures [30, 33•, 35]. Land use regression modeling was the most common exposure estimation procedure and was applied both in cohort studies and in population-based registry and health service studies, with many studies using this procedure for both $\mathrm{NO}_{2}$ or nitrogen oxides and particulate matter exposure estimation $[17,20 \bullet, 22$, 23, 24•, 26•, 28, 34•, 38• 39]. Two European studies employed air chemistry transport models, Weinmayr et al. for particulate matter estimation and Renzi et al. for ozone [38•, 41]. Renzi et al., one of the only two ozone studies, also used a dispersion modeling process for ozone estimation, while Jerrett et al. used the US Environmental Protection Agency Community Multiscale Air Quality System (an Eulerian chemical transport model) scaled to measured levels [24•,38•]. Liu et al. used the global chemical transport model TM5 to adjust for ozone with particulate matter less than $2.5 \mu \mathrm{g} / \mathrm{m}^{3}\left(\mathrm{PM}_{2.5}\right)$ estimates [31, 49]. Two studies used information only from air quality monitors for $\mathrm{PM}_{2.5}$ and for $\mathrm{NO}_{2}$, while another study used monitors in addition to a spatiotemporal model $[17,30,36]$. Only national or regional large population-based studies primarily relying on health service usage and ICD coding for diabetes assessment, employed aerosol optical depth satellite data to characterize $\mathrm{PM}_{2.5}$ exposures [20•, 25, 31, 36, 40]. A limitation of these methods is missingness in satellite observations due to cloud cover, though authors of the included studies accounted for missingness in their analyses in various ways. Only one study did not provide enough detail in the publication to identify the specific modeling process used for comparison to other studies [32].

As with all large ambient air pollution epidemiology studies, exposure estimation is necessary because personal exposure monitoring is neither available nor feasible. Thus, the spatial misalignment of monitors and study participants may lead to misclassification of exposure in various ways. There are of course limitations to each method of exposure estimation. In general, those that perform some form of spatial averaging are considered less susceptible to classical measurement error (referring to when instrument error or other non- 
representative biases are imputed) as opposed to Berkson-type errors (those which, while they may lead to imprecision in health effect estimates, do not cause bias in said estimates). Study findings did not appear to be clearly connected with particular types of exposure assessment for particular pollutants. Levels of exposure estimates produced by modeling techniques and nearest monitor imputation methods were generally demonstrative of higher levels for densely populated, heavily trafficked urban areas and lower levels for suburban/ rural areas. Overall, the strengths of these studies included generally well-considered and described exposure estimation methods with several studies using long time periods of available data. The consideration of dispersion factors and missingness of data as well as the inclusion of detailed information in publications is also necessary in future work, in order to evaluate the level of accuracy of the assessment, the sources considered, and whether background regional or local variation is included.

\section{Pollutants: Potential Biological Mechanisms and Study Findings}

The exact biological mechanisms by which ambient air pollution may be linked with diabetes have not been elucidated; however, experimental and epidemiologic research has investigated potential pathways. We present findings from the epidemiologic studies included in this review alongside this mechanistic work to enhance our examination of whether the literature suggests a relationship of type 2 diabetesrelated morbidity or mortality in adults with exposures to particulate matter, $\mathrm{NO}_{2}$ or nitrogen oxides, ozone, and other ambient air pollutants.

\section{Particulate Matter}

Experimental studies indicate that $\mathrm{PM}_{2.5}$ could act as a mediator of endothelial dysfunction and insulin resistance (IR). In one animal study, exposure to $\mathrm{PM}_{2.5}$ in combination with a high-fat diet was reported to increase fasting, postprandial glucose, insulin, and Homeostasis Model Assessment-IR similar to those observed with a high-fat diet over 24 weeks [50]. Elevated levels of tumor necrosis factor-alpha (TNF- $\alpha$ ), interleukin-6 (IL-6), resistin, and leptin levels were also observed indicating a proinflammatory IR-resistant state. In another study by Sun et al. [18], there was supportive evidence of abnormal insulin signaling. Exposure to $\mathrm{PM}_{2.5}$ in the absence of a high-fat diet has also been shown to increase HOMA-IR and postprandial glucose [51]. Limited epidemiology studies also report that exposure to ambient air pollution alters systemic biomarkers of inflammation [52, 53]. Another plausible biological mechanism includes endoplasmic reticulum (ER) stress [54]. In vitro studies have demonstrated that exposure to $\mathrm{PM}_{2.5}$ can induce ER stress, and this may represent a pathophysiologically relevant mechanism linking particulate matter exposure with hepatic insulin resistance. In other experimental studies, $\mathrm{PM}_{2.5}$ has been reported to decrease phosphorylation of Akt in the liver and skeletal muscle. Other changes observed included hepatic lipid deposition and decreased gluconeogenesis. In other research, $\mathrm{PM}_{2.5}$ exposure was associated with alterations in IR and glucose homeostasis $[55,56]$. Some experimental studies indicate that exposure to traffic-related fine and ultra fine particles (UFPs) and ozone trigger inflammation, oxidative stress, and biological pathways that promote metabolic IR [57-59].

Based on the long-term exposure epidemiology studies we reviewed, we found that particulate matter less than $10 \mu \mathrm{g} / \mathrm{m}^{3}$ $\left(\mathrm{PM}_{10}\right)$ exposures were positively associated with prevalent diabetes in two out of three studies (Fig. 1) and with incident diabetes in four of five studies (Fig. 1), with two prevalent studies and two of the incident studies reaching levels of statistical significance $[17,28,29 \bullet, 35,38 \bullet, 39,41]$. Two of the incidence studies with positive findings were conducted among women only [17, 29•]. No obvious consistent differences among studies were observed (e.g., mean population age or outcome assessment differences for those with stronger findings). Notably, a null association was reported for the study with the largest sample size and highest average exposure [38 $]$. Contrary to other studies which included urban and suburban/rural areas, this study was conducted in a population dense, heavily trafficked city, and also reported statistically significant positive associations with traffic-related pollutants. Incident diabetes was also statistically significantly associated with living in close proximity to a major road in two of three studies [19, 27, 35]. Differences among studies could be due to differences in $\mathrm{PM}_{10}$ composition and sources. Findings for $\mathrm{PM}_{2.5}$ exposures with diabetes prevalence and incidence were similar with the majority of studies showing positive associations, albeit with fewer studies reaching statistical significance (Fig. 1) $[17,25,30,31,36,40]$. Several studies were conducted among only women and showed positive associations [17, $23,29 \bullet]$. Both diabetes-related mortality studies of particulate exposures reported statistically significant increased risk associated with higher long-term exposures to $\mathrm{PM}_{2.5}[21,34 \bullet]$. No consistent pattern of differences among study methods, levels of exposure, or other factors we examined provide a clear explanation regarding the variations in findings. For UFPs, data are very limited in epidemiologic as well as animal studies $\left[20^{\bullet}\right]$.

\section{$\mathrm{NO}_{2}$ or Nitrogen Oxides}

$\mathrm{NO}_{2}$ or nitrogen oxides are gaseous pollutants that often serve as a proxy for traffic-related pollution. Epidemiologic studies 


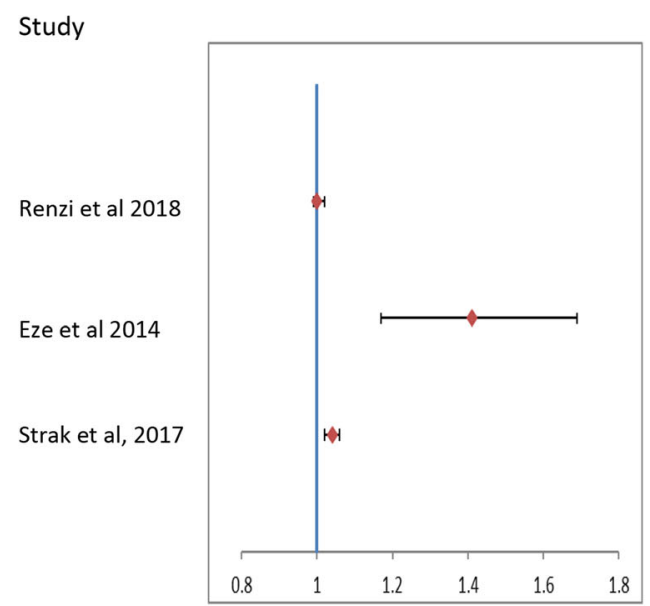

* Associations between Diabetes Prevalence and Chronic PM Exposures: Renzi et al 2018 and Eze et al 2014 for $10 \mu \mathrm{g} / \mathrm{m}^{3}$ change in $\mathrm{PM}_{10}$; IQR change for Strak et al, 2017

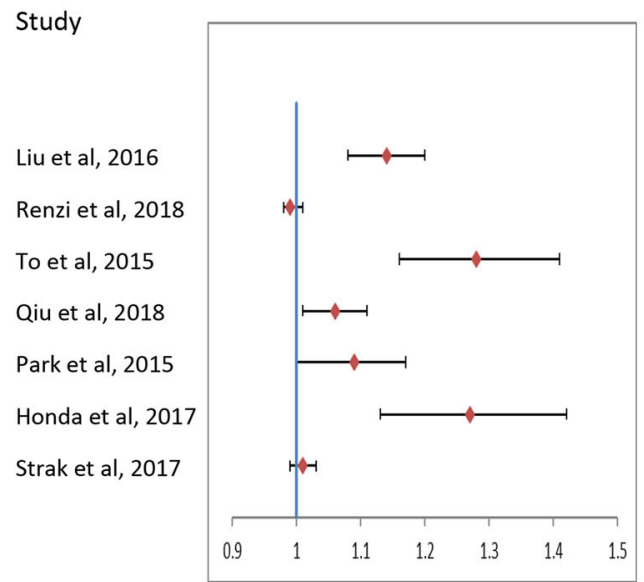

* Associations between Diabetes Prevalence and $\mathrm{PM}_{2.5}$ Exposures; Renzi et al $20185 \mathrm{\mu g} / \mathrm{m}^{3}$ change in $\mathrm{PM}_{2.5}$; To et al $201510 \mu \mathrm{g} / \mathrm{m}^{3}$ change in $\mathrm{PM}_{2.5} ;$ IQR change for others

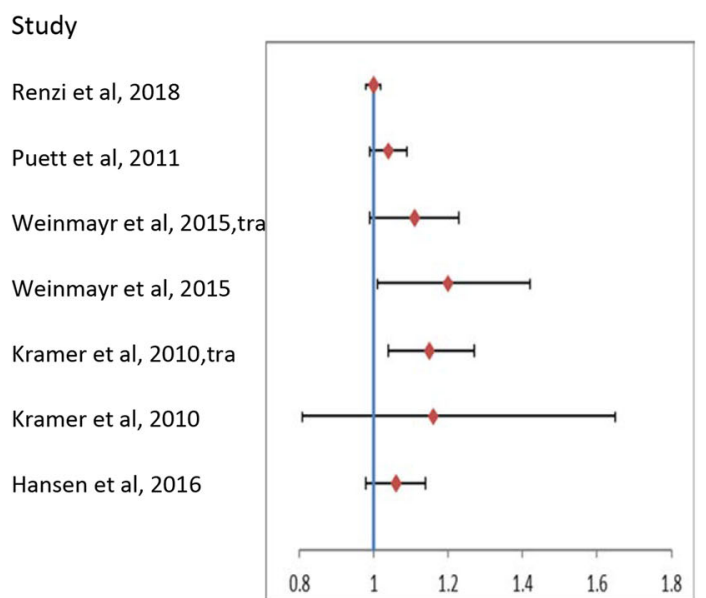

* Associations between Diabetes Incidence and Chronic PM $\mathrm{PM}_{10}$ Exposures; Renzi et al $201810 \mu \mathrm{g} / \mathrm{m}^{3}$ change in $\mathrm{PM}_{10}$; IQR change for all other studies; PMtra=traffic-specific PM

Study

Renzi et al, 2018

To et al, 2015

Chen et al, 2013

Puett et al, 2011

Park et al, 2015

Clark et al, 2017

Bai et al, 2018

Qiu et al, 2018

Weinmayer et al, 2015,tra

Weinmayer et al, 2015

Coogan et al, 2016

Kramer et al, 2010

Hansen et al, 2016

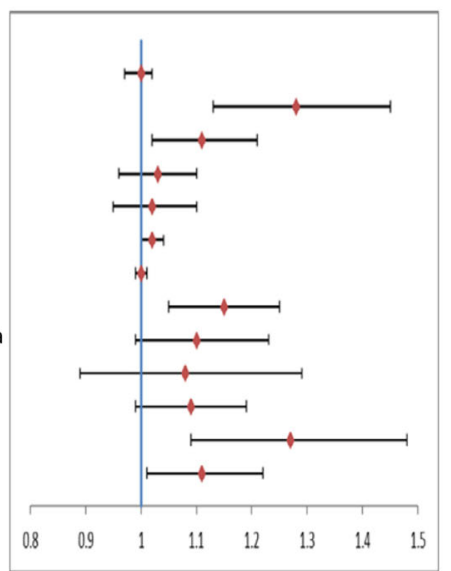

* Associations between Diabetes Incidence and PM ${ }_{2.5}$ Exposures; Renzi $5 \mu \mathrm{g} / \mathrm{m}^{3}$ change in $\mathrm{PM}_{2.5}$; To and Chen $10 \mu \mathrm{g} / \mathrm{m}^{3}$ change; IQR change for others; PMtra=traffic-specific PM

Fig. 1 Reported relative risks and 95\%confidence intervals for fully-adjusted models of associations between type 2 diabetes and particulate matter exposures

of associations and toxicologic studies providing evidence for pathophysiologic pathways for $\mathrm{NO}_{2}$ or nitrogen oxides are limited in part because these pollutants react with other pollutants, are correlated with other pollutants (positively and negatively), and are highly spatially variable; thus, both exposure estimation and distinguishing health outcomes specifically linked to $\mathrm{NO}_{2}$ or nitrogen oxides from other pollutants are particularly challenging $[24 \bullet, 60,61,62 \bullet \bullet]$. While underscoring the limitations and need for additional mechanistic and toxicology studies examining the long-term impacts of $\mathrm{NO}_{2}$ or nitrogen oxides, the review of evidence on health aspects of air pollution (REVIHAAP) Project: Technical Report suggests that current studies show some support for inflammation, airway hyperresponsiveness, and oxidative stress as potential pathophysiological mechanisms for adverse human health effects [62••]. Both oxidative stress and inflammation have been linked with insulin resistance and metabolic dysfunction.

Among the studies included in our review, positive associations were reported for all studies assessing long-term $\mathrm{NO}_{2}$ or nitrogen oxide exposures, indicators of traffic-related pollution, with diabetes prevalence (Fig. 2) [28, 30, 38•, 39]. Findings for diabetes incidence with $\mathrm{NO}_{2}$ were inconsistent, with about half of the studies reporting increasing risk with increasing exposures [17, 19, 20•, 29•]. An association between nitrogen oxides and incident diabetes was only supported by one (HR: 1.011, 95\%CI: 1.003-1.019) of three studies examining the relationship [29•, 33•, 38• $]$. One study explored diabetes-related mortality and $\mathrm{NO}_{2}$ exposures, reporting statistically significant increased risk with $\mathrm{NO}_{2}$ exposures in the year prior to death and an attenuated but positive association with longer-term $\mathrm{NO}_{2}$ exposures [37]. 


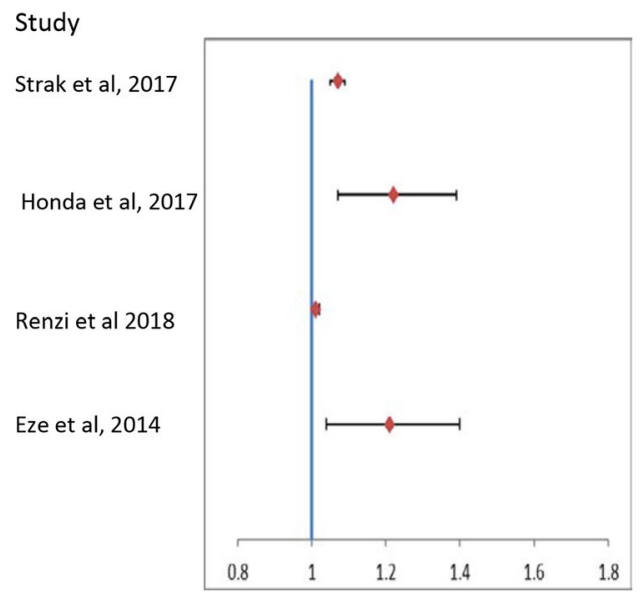

* Associations between Diabetes Prevalence and NO2 Exposures; Renzi et al, 2018 and Eze et al $201410 \mu \mathrm{g} / \mathrm{m}^{3}$ change in NO2; IQR change for others

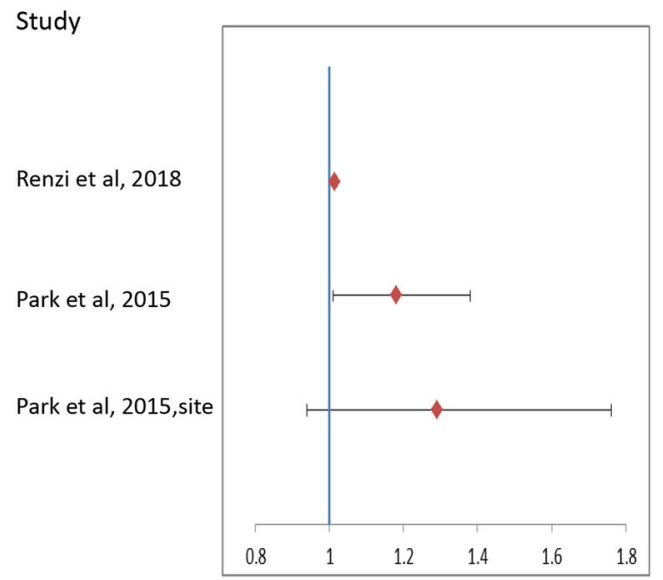

* Associations between Diabetes Prevalence and NOx Exposures Renzi et al $201820 \mu \mathrm{g} / \mathrm{m}^{3}$ change in NOx; IQR change for others ; site $=$ site adjusted model
Study

Renzi et al, 2018

Clark et al, 2017

Bai et al, 2018

Andersen et al, 2012

Kramer et al, 2010

Kramer et al, 2010 tra

Coogan et al, 2016

Hansen et al, 2016

O'Donovan et al, 2017

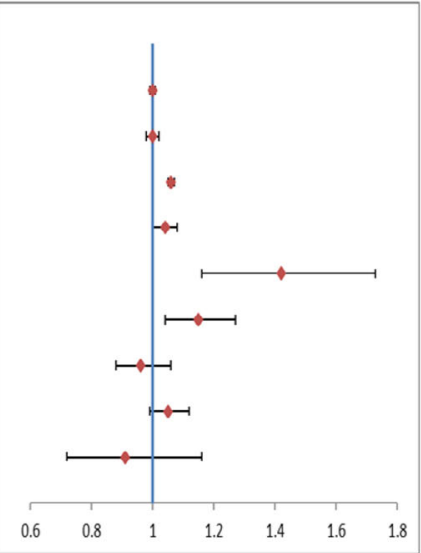

* Associations between Diabetes Incidence and NO2 Exposures; Renzi $5 \mathrm{\mu g} / \mathrm{m}^{3}$ change in No2; O'Donovan $10 \mu \mathrm{g} / \mathrm{m}^{3}$ change; IQR change for others; tra=traffic-specific NO

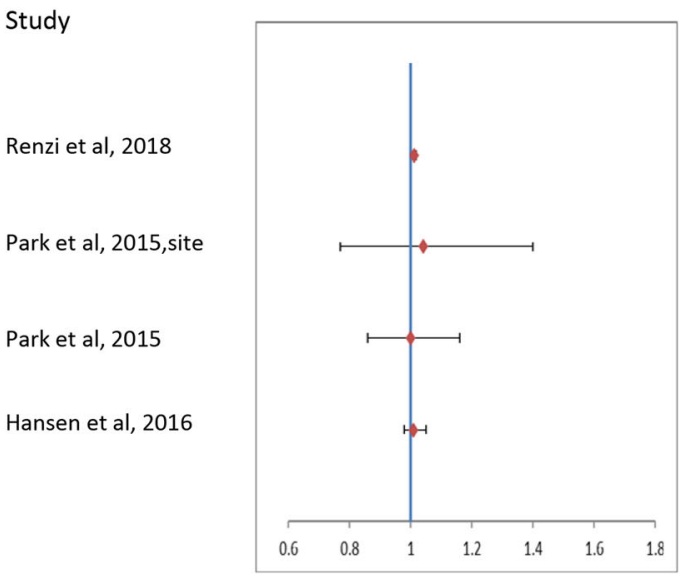

* Associations between Diabetes Incidence and NOx Exposures; 20 $\mu \mathrm{g} / \mathrm{m}^{3}$ change in NOx;IQR change for others; site=site adjusted model

Fig. 2 Reported relative risks and 95\%confidence intervals for fully-adjusted models of associations between type 2 diabetes and $\mathrm{NO}_{2}$ and $\mathrm{NO}_{\mathrm{x}}$, and exposures

\section{Ozone and Other Pollutants}

Ozone, formed by photochemical reactions on emissions from fossil fuel combustion and industrial and vehicular activities, has been linked to poorer fasting glucose and insulin levels, and HOMA indices among older adults, with stronger associations observed among those with a history of diabetes or increased susceptibility to oxidative stress $[63,64]$. One recent experimental study conducted by Vella et al. exposed rats to environmentally-relevant doses of ozone $(0.8 \mathrm{ppm})$ and found that exposure induced whole-body insulin resistance and oxidative stress with associated endoplasmic reticulum (ER) stress, c-Jun N-terminal kinase (JNK) activation, and disruption of insulin signaling in skeletal muscle [59]. The authors also reported that subchronic exposure to ozone $(0.25 \mathrm{ppm}, 12 \mathrm{~h} /$ day for 4 days) led to pulmonary inflammation, oxidative stress, and insulin resistance. Vella et al. suggest that production of lung mediators that induce oxidative stress and subsequent activation of JNK activation in skeletal muscle could disrupt insulin-induced signaling and glucose uptake [59].

Thus, although biologically plausible, only two studies examined long-term ozone exposures with prevalent diabetes, reporting a null association and a weak inverse association $[31,38 \bullet]$. Two studies reported statistically significant positive associations with incident diabetes. One study was conducted exclusively among women and the other study found stronger risks among women compared to men $[24 \bullet, 38 \bullet]$.

\section{Susceptible Subpopulations}

Differences in human susceptibility to environmental exposures can be related to comorbid disease; demographic or anthropometric characteristics; genetics; race and ethnicity; 
lifestyle, behaviors, and socioeconomic position. In general, the studies included in our review also considered whether differences in effects varied based on age group, sex, socioeconomic status, smoking, alcohol consumption, diet, comorbidities, and body mass index (BMI). Overall, consistent evidence of effect modification by any one of these factors or for any particular pollutant exposures/diabetes outcomes was limited, with the possible exception of differences based on age and sex.

Most studies examining age group differences reported none; however, a few studies reported observing higher risks among participants under 50 years of age compared to older groups for incident diabetes associated with exposure to UFPs, $\mathrm{PM}_{10}$ and with ozone $[20 \bullet, 25,38 \bullet]$. These findings are noteworthy as type 2 diabetes incidence tends to increase with age, and recently, type 2 diabetes is becoming more common among youth [65-67].

Some studies also reported differences by sex, with few interactions statistically significant. Weinmayer et al. reported stronger risks among men for incident diabetes associated with $\mathrm{PM}_{10}$ and $\mathrm{PM}_{2.5}$ exposures and Puett et al. for $\mathrm{PM}_{2.5}$, while others reported stronger associations with $\mathrm{PM}_{2.5}$ for women [25, 35, 36, 41]. Puett et al. reported a strong positive association between living within $50 \mathrm{~m}$ of a heavily traveled road compared to living more than $200 \mathrm{~m}$ from the road for women, but the same finding was not true for men [35]. Studies also reported stronger associations among women compared to men for incident or prevalent diabetes with higher $\mathrm{NO}_{2}$ or nitrogen oxide exposures [19, 20•, 27, 33•].

\section{Areas for Further Research}

The implications of findings from the studies we examined are similar to those raised in previous reviews [68-71]. Welldesigned epidemiologic studies of type 2 diabetes and longterm ambient air pollution exposures with rigorous methods and exposure assessment can inform clinical treatment practices and air quality regulatory guidelines. Large populationbased studies with administrative data and cohort studies with more detailed information, including biomarkers and periodic follow-ups, are still needed to address inconsistent findings and areas with limited information, such as potential effect modification by sex and age for certain pollutants and associations in underrepresented groups (e.g., racial/ethnic minorities). Challenges may exist with regard to air pollution modeling, but additional well-designed and clearly reported studies in areas of high pollution and diabetes prevalence/incidence are needed to provide additional information about etiology and group differences. With respect to gaining further insights regarding higher risks among younger age groups, future studies will need careful consideration of age range inclusions and case definitions in order to gain information about type 2 diabetes in younger populations while avoiding potential misclassification by including type 1 diabetes cases, which is more common under age 30. Approximately half of the studies included in this review reported a mean/median age (typically at baseline) between 50 and 65 years with additional studies focused on populations with mean/median ages 70 and older, limiting numbers of younger study participants $[17,19$, 20•, 25, 26•, 28, 29•, 30-32, 33•, 35-37, 40, 41•].

Administrative data and well-characterized cohort data that include biomarkers, symptomology, and medical treatment can enhance sensitivity as well as specificity of diabetes diagnosis. This is critical for ensuring type 2 diabetes outcome accuracy to reduce potential misclassification and bias in main association studies, potentially explaining some of the inconsistencies observed in findings across the studies reviewed. For example, in a study assessing the importance of diabetes case definitions used in epidemiologic research, Bielinski and colleagues reported that the magnitude of associations between known risk factors and diabetes varied depending on the criteria that were selected to identify diabetes cases [48]. Some studies included in this review addressed this issue by reporting results from sensitivity analyses conducted with multiple diabetes case assessment definitions [28, 35, 37].

In addition, better sensitivity and more comprehensive data are key for mechanistic studies, another area warranting further research. For example, studies have reported that statins, a class of drugs often prescribed by doctors to help lower cholesterol levels, reduced the risk of cardiovascular outcomes associated with particulate matter exposures, particularly among people with diabetes; a finding that has possible implications for diabetes studies if statin use in the population under study is unknown [72, 73]. Some of the cohort studies with more comprehensive biomarker data that were included in this review have embarked on initial mechanistic research. For example, Kramer and colleagues also examined the influence of an inflammatory biomarker on the relationship of incident diabetes and traffic-related long-term air pollutant exposures [17]. Eze et al. followed their main association study with mechanistically focused research, reporting an association between $\mathrm{PM}_{10}$ exposures and diabetes among individuals with pro-inflammatory candidate gene polymorphisms, providing further evidence for the inflammatory pathway [30, 74].

With regard to specific pollutants, epidemiologic data are very limited with respect to ultrafine particulates and ozone exposures. Prevalence and incidence association differences also suggest further research is needed with $\mathrm{NO}_{2}$ and $\mathrm{NOx}$ exposures. Toxicological research on long-term $\mathrm{NO}_{2}$ and nitrogen oxides exposures is severely limited. In epidemiologic studies, challenges clearly exist for distinguishing $\mathrm{NO}_{2}$ or nitrogen oxides impacts from those of correlated pollutants. For example, Jerrett et al. examined and discussed the tradeoff between ozone and $\mathrm{NO}_{2}$ levels (i.e., when one pollutant level is high, the other is low) [24•]. Thus, the more highly concentrated pollutant might mask the association with diabetes by 
the other pollutant. The REVIHAAP Report also described that $\mathrm{NO}_{2}$ is linked with particles and suggested that studies could include $\mathrm{NO}_{2}$ and a particle mass indicator (e.g., ultrafines or black carbon) $[62 \cdot \bullet]$.

Some of the studies included in this review investigated the impact of co-pollutants in predictive models compared with single pollutant models to demonstrate independent effects; however, challenges exist when these pollutants are correlated [e.g., 24•, 31, 35]. Most co-pollutants were other ambient air pollutants; however, noise is an important co-pollutant that has been linked with adverse health effects. The role of noise pollution is difficult to distinguish from air pollution. In this review, we included relevant findings from one study that focused primarily on noise, with air pollution treated as a copollutant [26•]. The authors reported that noise was associated with incident diabetes independent of air pollution, while the reverse situation was not observed. Findings from this and other studies in this small but growing body of work will provide important information with respect to whether each type of pollutant differentially impacts certain aspects of the diabetes pathway or certain subpopulations [75].

Studies in this review and rigorous methodological studies addressing the needs described above are important for supporting further policy and translational work in risk assessment and cost-benefit analyses which can more directly support air quality policies. Previous approaches for quantitatively incorporating population vulnerability and susceptibility in risk assessment have accounted for differences in baseline heath status using measures such as cause-specific death rates, hospital/emergency department visit rates, disease prevalence rates, and/or pollutant-specific differential relative risk estimates, which may serve as models for future diabetes and air pollution work [76-80, 81••].

\section{Conclusions}

In summary, the studies included in this review generally employed rigorous methodology with large sample sizes, appropriate study designs to maximize available cohort study or administrative data sources, and exposure modeling that accounted for air pollution dispersion factors. Overall, most studies for each pollutant/outcome reported increased risks associated with increased exposures; however, findings were not uniformly positive nor statistically significant. Further research is needed to provide a more comprehensive understanding for clinical treatment and air quality regulatory guidelines. Current research is particularly limited regarding the biological mechanisms involved, examining the relationship between ozone and diabetes, distinguishing $\mathrm{NO}_{2}$ or nitrogen oxides effects from those of other pollutants, and identification of potential subpopulations with greater susceptibility for select pollutant exposures.

\section{Compliance with Ethical Standards}

Conflict of Interest Robin Puett received an honorarium and travel expenses to present part of this work at the Health Effects Institute Conference in April 2018. Lesliam Quiros-Alcala, Jessica MontresorLopez, Nedelina Tchangalova, Anindita Dutta, Devon Payne-Sturges and Jeff Yanosky each declare no potential conflicts of interest.

Human and Animal Rights and Informed Consent This article does not contain any studies with human or animal subjects performed by any of the authors.

Publisher's Note Springer Nature remains neutral with regard to jurisdictional claims in published maps and institutional affiliations.

\section{References}

Papers of particular interest, published recently, have been highlighted as:

- Of importance

•• Of major importance

1.• World Health Organization. Ambient (outdoor) air quality and health. Geneva World Health Organ. 2018 [cited 2018 Nov 4]. Available from: http://www.who.int/news-room/fact-sheets/detail/ ambient-(outdoor)-air-quality-and-health. This report is significant for publicizing the global impacts of ambient air pollution on health, particularly for children and low and middle income countries.

2. World Health Organization. Ambient air pollution: health impacts. Geneva World Health Organ. [cited 2018 Nov 2]. Available from: http://www.who.int/airpollution/ambient/health-impacts/en/.

3. Hamra GB, Guha N, Cohen A, Laden F, Raaschou-Nielsen O, Samet JM, et al. Outdoor particulate matter exposure and lung cancer: a systematic review and meta-analysis. Environ Health Perspect. 2014;122:906-11.

4. Pope CA, Dockery DW. Health effects of fine particulate air pollution: lines that connect. J Air Waste Manag Assoc 1995. 2006;56: 709-42.

5. Suades-González E, Gascon M, Guxens M, Sunyer J. Air pollution and neuropsychological development: a review of the latest evidence. Endocrinology. 2015;156:3473-82.

6. Westergaard N, Gehring U, Slama R, Pedersen M. Ambient air pollution and low birth weight - are some women more vulnerable than others? Environ Int. 2017;104:146-54.

7. Dubowsky SD, Suh H, Schwartz J, Coull BA, Gold DR. Diabetes, obesity, and hypertension may enhance associations between air pollution and markers of systemic inflammation. Environ Health Perspect. 2006;114:992-8.

8.• U.S. Environmental Protection Agency. Integrated Science Assessment (ISA) for particulate matter (external review draft) [Internet]. Wash. DC US Environemntal Prot. Agency EPA600R18179. 2018 [cited 2018 Nov 4]. Available from: https://cfpub.epa. gov/ncea/isa/recordisplay.cfm?deid $=341593$. This synthesis of science is prepared as part of the review for National Ambient Air Quality Standards in the US as mandated by the Clean Air Act. It is open for public comment until December 11, 2018.

9. Zanobetti A, Schwartz J. Cardiovascular damage by airborne particles: are diabetics more susceptible? Epidemiol Camb Mass. 2002;13:588-92. 
10. Zeka A, Zanobetti A, Schwartz J. Individual-level modifiers of the effects of particulate matter on daily mortality. Am J Epidemiol. 2006;163:849-59.

11. Bowe B, Xie Y, Li T, Yan Y, Xian H, Al-Aly Z. The 2016 global and national burden of diabetes mellitus attributable to PM2.5 air pollution. Lancet Planet Health. 2018;2:e301-12.

12. O'Donovan G, Cadena-Gaitán C. Air pollution and diabetes: it's time to get active! Lancet Planet Health. 2018;2:e287-8.

13. Bommer C, Sagalova V, Heesemann E, Manne-Goehler J, Atun R, Bärnighausen T, et al. Global economic burden of diabetes in adults: projections from 2015 to 2030 . Diabetes Care. $2018 ; \mathrm{dc} 171962$.

14. National Institute of Diabetes and Digestive and Kidney Diseases. Type 2 diabetes. Bethesda MD NIDDK. [cited 2018 Nov 5]. Available from: https:/www.niddk.nih.gov/health-information/ diabetes/overview/what-is-diabetes/type-2-diabetes.

15. Kempf K, Rathmann W, Herder C. Impaired glucose regulation and type 2 diabetes in children and adolescents. Diabetes Metab Res Rev. 2008;24:427-37.

16. Kolb H, Mandrup-Poulsen T. An immune origin of type 2 diabetes? Diabetologia. 2005;48:1038-50.

17. Krämer U, Herder C, Sugiri D, Strassburger K, Schikowski T, Ranft U, et al. Traffic-related air pollution and incident type 2 diabetes: results from the SALIA cohort study. Environ Health Perspect. 2010;118:1273-9.

18. Sun Q, Yue P, Deiuliis JA, Lumeng CN, Kampfrath T, Mikolaj MB, et al. Ambient air pollution exaggerates adipose inflammation and insulin resistance in a mouse model of diet-induced obesity. Circulation. 2009;119:538-46.

19. Andersen ZJ, Raaschou-Nielsen O, Ketzel M, Jensen SS, Hvidberg M, Loft S, et al. Diabetes incidence and long-term exposure to air pollution: a cohort study. Diabetes Care. 2012;35:92-8.

20. Bai L, Chen H, Hatzopoulou M, Jerrett M, Kwong JC, Burnett RT, et al. Exposure to ambient ultrafine particles and nitrogen dioxide and incident hypertension and diabetes. Epidemiol Camb Mass. 2018;29:323-32 This large population-based study in Toronto adds to the dearth of information on long-term ultrafine particulate exposures and incident diabetes. The authors reported increasing risk with increasing exposures.

21. Brook RD, Cakmak S, Turner MC, Brook JR, Crouse DL, Peters PA, et al. Long-term fine particulate matter exposure and mortality from diabetes in Canada. Diabetes Care. 2013;36:3313-20.

22. Coogan PF, White LF, Yu J, Burnett RT, Marshall JD, Seto E, et al. Long term exposure to $\mathrm{NO}_{2}$ and diabetes incidence in the Black Women's Health Study. Environ Res. 2016;148:360-6 This publication, along with $[20,22]$ examine a population at high risk for cardiometabolic morbidity that has traditionally been underrepresented in US studies of ambient air pollution.

23. Coogan PF, White LF, Yu J, Burnett RT, Seto E, Brook RD, et al. PM2.5 and diabetes and hypertension incidence in the black Women's health study. Epidemiol Camb Mass. 2016;27:202-10.

24. Jerrett M, Brook R, White LF, Burnett RT, Yu J, Su J, et al. Ambient ozone and incident diabetes: a prospective analysis in a large cohort of African American women. Environ Int. 2017;102:42-7 One of two studies we identified on long-term ozone and type 2 diabetes. A significant risk of incident diabetes was associated with increases in ozone exposures.

25. Chen H, Burnett RT, Kwong JC, Villeneuve PJ, Goldberg MS, Brook RD, et al. Risk of incident diabetes in relation to long-term exposure to fine particulate matter in Ontario, Canada. Environ Health Perspect. 2013;121:804-10.

26. Clark C, Sbihi H, Tamburic L, Brauer M, Frank LD, Davies HW. Association of long-term exposure to transportation noise and traffic-related air pollution with the incidence of diabetes: a prospective cohort study. Environ Health Perspect. 2017;125:087025 This study examined air pollution and noise as co-pollutants, reporting noise as an independent predictor of incident diabetes and greenness index as protective, and no association with PM, black carbon or nitrogen oxides when adjusted for noise.

27. Dijkema MBA, Mallant SF, Gehring U, van den Hurk K, Alssema $\mathrm{M}$, van Strien RT, et al. Long-term exposure to traffic-related air pollution and type 2 diabetes prevalence in a cross-sectional screening-study in the Netherlands. Environ Health Glob Access Sci Source. 2011;10:76

28. Eze IC, Schaffner E, Fischer E, Schikowski T, Adam M, Imboden $\mathrm{M}$, et al. Long-term air pollution exposure and diabetes in a population-based Swiss cohort. Environ Int. 2014;70:95-105.

29. Hansen AB, Ravnskjær L, Loft S, Andersen KK, Bräuner EV, Baastrup R, et al. Long-term exposure to fine particulate matter and incidence of diabetes in the Danish Nurse Cohort. Environ Int. 2016;91:243-50 This is one of very few studies on longterm nitrogen oxides exposures and diabetes. This study of a large cohort of women found a weak positive association between incident diabetes and nitrogen oxides and a significant increase with $\mathbf{P M}_{2.5}$ exposures.

30. Honda T, Pun VC, Manjourides J, Suh H. Associations between long-term exposure to air pollution, glycosylated hemoglobin and diabetes. Int J Hyg Environ Health. 2017;220:1124-32.

31. Liu C, Yang C, Zhao Y, Ma Z, Bi J, Liu Y, et al. Associations between long-term exposure to ambient particulate air pollution and type 2 diabetes prevalence, blood glucose and glycosylated hemoglobin levels in China. Environ Int. 2016;92-93:416-21.

32. O'Donovan G, Chudasama Y, Grocock S, Leigh R, Dalton AM, Gray LJ, et al. The association between air pollution and type 2 diabetes in a large cross-sectional study in Leicester: the CHAMPIONS study. Environ Int. 2017;104:41-7.

33. Park SK, Adar SD, O’Neill MS, Auchincloss AH, Szpiro A, Bertoni AG, et al. Long-term exposure to air pollution and type 2 diabetes mellitus in a multiethnic cohort. Am J Epidemiol. 2015;181:327-36 This diverse cohort includes groups typically underrepresented in air pollution epidemiology studies and was one of the few studies to examine the association of incident diabetes with nitrogen oxides, finding a weak positive association when adjusting for site.

34. Pope CA, Turner MC, Burnett RT, Jerrett M, Gapstur SM, Diver WR, et al. Relationships between fine particulate air pollution, cardiometabolic disorders, and cardiovascular mortality. Circ Res. 2015;116:108-15 One of few studies examining long-term exposures to $\mathbf{P M}_{2.5}$ and cardiometabolic disease mortality. The study conducted in a large cohort in the US, found a significant positive association.

35. Puett RC, Hart JE, Schwartz J, Hu FB, Liese AD, Laden F. Are particulate matter exposures associated with risk of type 2 diabetes? Environ Health Perspect. 2011;119:384-9.

36. Qiu H, Schooling CM, Sun S, Tsang H, Yang Y, Lee RS, et al. Long-term exposure to fine particulate matter air pollution and type 2 diabetes mellitus in elderly: a cohort study in Hong Kong. Environ Int. 2018;113:350-6.

37. Raaschou-Nielsen O, Sørensen M, Ketzel M, Hertel O, Loft S, Tjønneland A, et al. Long-term exposure to traffic-related air pollution and diabetes-associated mortality: a cohort study. Diabetologia. 2013;56:36-46.

38. Renzi M, Cerza F, Gariazzo C, Agabiti N, Cascini S, Di Domenicantonio R, et al. Air pollution and occurrence of type 2 diabetes in a large cohort study. Environ Int. 2018;112:68-76 One of very few studies on long-term ozone and nitrogen oxides exposures with incident diabetes. The large population-based study conducted in Rome had some of the highest exposure concentrations but found no association with PM or NO2 but significant positive associations with nitrogen oxides and ozone. 
39. Strak M, Janssen N, Beelen R, Schmitz O, Vaartjes I, Karssenberg $\mathrm{D}$, et al. Long-term exposure to particulate matter, $\mathrm{NO}_{2}$ and the oxidative potential of particulates and diabetes prevalence in a large national health survey. Environ Int. 2017;108:228-36.

40. To T, Zhu J, Villeneuve PJ, Simatovic J, Feldman L, Gao C, et al. Chronic disease prevalence in women and air pollution: a 30-year longitudinal cohort study. Environ Int. 2015;80:26-32.

41. Weinmayr G, Hennig F, Fuks K, Nonnemacher M, Jakobs H, Möhlenkamp S, et al. Long-term exposure to fine particulate matter and incidence of type 2 diabetes mellitus in a cohort study: effects of total and traffic-specific air pollution. Environ Health. 2015 [cited 2018 Nov 4]; 14. Available from: https://www.ncbi.nlm.nih.gov/ pmc/articles/PMC4479324/.

42. World Health Organization. Definition and diagnosis of diabetes mellitus and intermediate hyperglycaemia. Geneva World Health Organ. 2006 [cited 2018 Nov 4]. Available from: https://www. who.int/diabetes/publications/diagnosis_diabetes2006/en/.

43. American Diabetes Association. Diagnosis and classification of diabetes mellitus. Diabetes Care. 2014;37:S81-90.

44. Beagley J, Guariguata L, Weil C, Motala AA. Global estimates of undiagnosed diabetes in adults. Diabetes Res Clin Pract. 2014;103: 150-60.

45. Cho NH, Shaw JE, Karuranga S, Huang Y, da Rocha Fernandes JD, Ohlrogge AW, et al. IDF diabetes atlas: global estimates of diabetes prevalence for 2017 and projections for 2045. Diabetes Res Clin Pract. 2018;138:271-81.

46. Ning M, Zhang Q, Yang M. Comparison of self-reported and biomedical data on hypertension and diabetes: findings from the China health and retirement longitudinal study (CHARLS). BMJ Open. 2016;6:e09836.

47. Schneider ALC, Pankow JS, Heiss G, Selvin E. Validity and reliability of self-reported diabetes in the atherosclerosis risk in communities study. Am J Epidemiol. 2012;176:738-43.

48. Bielinski SJ, Pankow JS, Rasmussen-Torvik LJ, Bailey K, Li M, Selvin E, et al. Strength of association for incident diabetes risk factors according to diabetes case definitions. Am J Epidemiol. 2012;175:466-72.

49. Brauer M, Freedman G, Frostad J, van Donkelaar A, Martin RV, Dentener F, et al. Ambient air pollution exposure estimation for the global burden of disease 2013. Environ Sci Technol. 2016;50:7988.

50. Laing S, Wang G, Briazova T, Zhang C, Wang A, Zheng Z, et al. Airborne particulate matter selectively activates endoplasmic reticulum stress response in the lung and liver tissues. Am J Physiol Cell Physiol. 2010;299:C736-49.

51. Xu X, Yavar Z, Verdin M, Ying Z, Mihai G, Kampfrath T, et al. Effect of early particulate air pollution exposure on obesity in mice: role of p47phox. Arterioscler Thromb Vasc Biol. 2010;30:2518-27.

52. Rich DQ, Kipen HM, Huang W, Wang G, Wang Y, Zhu P, et al. Association between changes in air pollution levels during the Beijing olympics and biomarkers of inflammation and thrombosis in healthy young adults. JAMA J Am Med Assoc. 2012;307:206878 .

53. Rückerl R, Hampel R, Breitner S, Cyrys J, Kraus U, Carter J, et al. Associations between ambient air pollution and blood markers of inflammation and coagulation/fibrinolysis in susceptible populations. Environ Int. 2014;70:32-49.

54. Walter P, Ron D. The unfolded protein response: from stress pathway to homeostatic regulation. Science. 2011;334:1081-6.

55. Xu X, Liu C, Xu Z, Tzan K, Zhong M, Wang A, et al. Long-term exposure to ambient fine particulate pollution induces insulin resistance and mitochondrial alteration in adipose tissue. Toxicol Sci Off J Soc Toxicol. 2011;124:88-98.

56. Zheng Z, Xu X, Zhang X, Wang A, Zhang C, Hüttemann M, et al. Exposure to ambient particulate matter induces a NASH-like phenotype and impairs hepatic glucose metabolism in an animal model. J Hepatol. 2013;58:148-54.

57. Brook RD, Xu X, Bard RL, Dvonch JT, Morishita M, Kaciroti N, et al. Reduced metabolic insulin sensitivity following sub-acute exposures to low levels of ambient fine particulate matter air pollution. Sci Total Environ. 2013;448:66-71.

58. Rajagopalan S, Brook RD. Air pollution and type 2 diabetes: mechanistic insights. Diabetes. 2012;61:3037-45.

59. Vella RE, Pillon NJ, Zarrouki B, Croze ML, Koppe L, Guichardant $\mathrm{M}$, et al. Ozone exposure triggers insulin resistance through muscle c-Jun N-terminal kinase activation. Diabetes. 2015;64:1011-24.

60. Faustini A, Rapp R, Forastiere F. Nitrogen dioxide and mortality: review and meta-analysis of long-term studies. Eur Respir J. 2014;44:744-53.

61. Hoek G, Krishnan RM, Beelen R, Peters A, Ostro B, Brunekreef B, et al. Long-term air pollution exposure and cardio- respiratory mortality: a review. Environ Health Glob Access Sci Source. 2013;12:43.

62.• World Health Organization Regional Office for Europe. Review of evidence on health aspects of air pollution - REVIHAAP Project: Technical Report. Copenhagen: WHO Regional Office for Europe; 2013 [cited 2018 Nov 5]. Available from: http://www.ncbi.nlm.nih. gov/books/NBK361805/. A large group of scientists provided this comprehensive review for the purpose of reviewing European air pollution policies and health impacts.

63. Finlayson-Pitts BJ, Pitts JN. Tropospheric air pollution: ozone, airborne toxics, polycyclic aromatic hydrocarbons, and particles. Science. 1997;276:1045-52.

64. Kim JH, Hong Y-C. GSTM1, GSTT1, and GSTP1 polymorphisms and associations between air pollutants and markers of insulin resistance in elderly Koreans. Environ Health Perspect. 2012;120: 1378-84.

65. Centers for Disease Control and Prevention. National diabetes statistics report: estimates of diabetes and its burden in the United States. 2018 [cited 2018 Nov 5]. Available from: https://www.cdc. gov/diabetes/data/statistics/statistics-report.html.

66. Monesi L, Baviera M, Marzona I, Avanzini F, Monesi G, Nobili A, et al. Prevalence, incidence and mortality of diagnosed diabetes: evidence from an Italian population-based study. Diabet Med J Br Diabet Assoc. 2012;29:385-92.

67. Ubink-Veltmaat LJ, Bilo HJG, Groenier KH, Houweling ST, Rischen RO, Meyboom-de JB. Prevalence, incidence and mortality of type 2 diabetes mellitus revisited: a prospective population-based study in the Netherlands (ZODIAC-1). Eur J Epidemiol. 2003;18: 793-800.

68. Eze IC, Hemkens LG, Bucher HC, Hoffmann B, Schindler C, Künzli N, et al. Association between ambient air pollution and diabetes mellitus in Europe and North America: systematic review and meta-analysis. Environ Health Perspect. 2015;123:381-9.

69. Rao X, Montresor-Lopez J, Puett R, Rajagopalan S, Brook RD. Ambient air pollution: an emerging risk factor for diabetes mellitus. Curr Diab Rep. 2015;15:603.

70. Thiering E, Heinrich J. Epidemiology of air pollution and diabetes. Trends Endocrinol Metab TEM. 2015;26:384-94.

71. Wang B, Xu D, Jing Z, Liu D, Yan S, Wang Y. Effect of long-term exposure to air pollution on type 2 diabetes mellitus risk: a systemic review and meta-analysis of cohort studies. Eur J Endocrinol. 2014; 171:R173-82.

72. O'Neill MS, Veves A, Sarnat JA, Zanobetti A, Gold DR, Economides PA, et al. Air pollution and inflammation in type 2 diabetes: a mechanism for susceptibility. Occup Environ Med. 2007;64:373-9.

73. Ostro B, Malig B, Broadwin R, Basu R, Gold EB, Bromberger JT, et al. Chronic PM2.5 exposure and inflammation: determining sensitive subgroups in mid-life women. Environ Res. 2014;132:168-75.

74. Eze IC, Imboden M, Kumar A, Adam M, von Eckardstein A, Stolz $\mathrm{D}$, et al. A common functional variant on the pro-inflammatory 
Interleukin-6 gene may modify the association between long-term PM10 exposure and diabetes. Environ Health Glob Access Sci Source. 2016;15:39.

75. Zare Sakhvidi MJ, Zare Sakhvidi F, Mehrparvar AH, Foraster M, Dadvand P. Association between noise exposure and diabetes: a systematic review and meta-analysis. Environ Res. 2018;166: 647-57.

76. Chari R, Burke TA, White RH, Fox MA. Integrating susceptibility into environmental policy: an analysis of the national ambient air quality standard for lead. Int J Environ Res Public Health. 2012;9: 1077-96.

77. Cullen AC, Corrales MA, Kramer CB, Faustman EM. The application of genetic information for regulatory standard setting under the clean air act: a decision-analytic approach. Risk Anal. 2008;28: 877-90.

78. Fann N, Roman HA, Fulcher CM, Gentile MA, Hubbell BJ, Wesson K, et al. Maximizing health benefits and minimizing inequality: incorporating local-scale data in the design and evaluation of air quality policies. Risk Anal Off Publ Soc Risk Anal. 2011;31: 908-22.

79. Levy JI, Greco SL, Spengler JD. The importance of population susceptibility for air pollution risk assessment: a case study of power plants near Washington. DC Environ Health Perspect. 2002;110: 1253-60.

80. Schwartz J, Bellinger D, Glass T. Expanding the scope of risk assessment: methods of studying differential vulnerability and susceptibility. Am J Public Health. 2011;101(Suppl 1):S102-9.

81.• U.S. Environmental Protection Agency. Regulatory impact analysis for the proposed revisions to the national ambient air quality standards for particulate matter. Wash. DC US Environemntal Prot. Agency EPA-452R-12-003. 2012 [cited 2018 Nov 4]. Available from: https://www3.epa.gov/ttnecas 1/regdata/RIAs/ PMRIACombinedFile_Bookmarked.pdf. As part of the the US review of national air pollution regulations, this report provides benefits and costs, monetary and non-monetary. 